\title{
ESTUDO DO IMPACTO NO TRÂNSITO EM DOIS TRECHOS DA VIA RÁPIDA DO EIXO ESTRUTURAL DE CURITIBA PELA IMPLANTAÇÁO DE FAIXAS EXCLUSIVAS UTILIZANDO SIMULADORES DE TRÁFEGO
}

\author{
M. A. P. Bernardinis ${ }^{1}$; T. A. Pianezzer'² ${ }^{2}$ G. L. Monich ${ }^{3}$ \\ ${ }^{1}$ Universidade Federal do Paraná, Centro Politécnico, Departamento de Transportes, bloco V, Jd. das Américas \\ ${ }^{2}$ Pós-Graduando da Universidade Federal de Santa Catarina, Campus Trindade, Departamento de Gestão \\ Territorial e Engenharia de Transportes \\ ${ }^{3}$ Graduada pela Universidade Federal do Paraná no curso de Engenharia Civil, Centro Politécnico, \\ Jd. das Américas \\ profmarcia.map@gmail.com ${ }^{1}$,tiago.pianezzer@gmail.com², gabrielamonich@gmail.com ${ }^{3}$
}

\begin{abstract}
Resumo: Esse artigo visa discutir a faixa exclusiva de ônibus como uma solução para o problema do trânsito e a utilização de softwares de tráfego para análises dela. Com uma coleta de dados de tráfego na cidade de Curitiba e com a utilização do software Vissim, observa-se a atual situação do trânsito da cidade e simula-se um cenário com essas faixas exclusivas em uma de suas vias. Foi comparado o número de veículos que transitou nessas vias e o nível de serviço dos cruzamentos antes e depois da simulação e observou-se uma retenção de veículos particulares de até $7,94 \%$ e uma piora nos níveis de serviço dos cruzamentos. Também foi possível concluir com o software que uma alternativa de melhora voltada para o trânsito de veículos coletivo não é necessariamente benéfica para esses veículos e que os softwares de tráfego são ferramentas essenciais para chegar à essas conclusôes.
\end{abstract}

Palavras-chave: Transporte público, Faixa exclusiva, Simulador de tráfego.

Abstract: This article aims to discuss the exclusive bus route as a solution to the traffic problem and the use of traffic software for its analysis. With a data collection of traffic in the city of Curitiba and using the software Vissim, we observe the current situation of city traffic and simulate a scenario with these exclusive lanes in one of its tracks. The number of vehicles transported in these routes and the service level of the crossings before and after the simulation were compared and a private vehicles retention of up to $7.94 \%$ and a decrease in service levels of the crossings were observed. It has also been possible to conclude from the software that an alternative to collective vehicle traffic improvement is not necessarily beneficial to these vehicles and that traffic software is an essential tool to reach these conclusions.

Keywords: Public transport, Exclusive lane, Traffic simulator. 


\section{Introduçáo}

A situação atual do tráfego não permite que se coloquem mais carros em circulação nas vias urbanas do país, visto que as cidades não tem mais para onde expandir suas malhas viárias para acomodá-los. Além do que, devido aos diversos problemas ambientais que as cidades enfrentam atualmente, é necessária uma redução no incentivo do uso de modais de transporte individuais. Assim, os benefícios da incorporação de uma mobilidade urbana sustentável vão além da área ambiental, proporcionando melhoria também nas áreas social, econômica e da saúde. De acordo com o [1], o desenvolvimento de uma mobilidade urbana sustentável melhora a qualidade de vida através da criação de espaços públicos mais atrativos, melhorando assim a segurança viária e diminuição da poluição sonora e do ar.

Junto disso, nota-se a necessidade da troca de uma cultura voltada a veículos individuais para uma mobilidade pensada de forma mais sustentável. Atualmente até já são notados movimentos sociais em prol de novas formas de mobilidade. Entretanto, vale ressaltar que intervençóes nesse setor podem causar mais prejuízos do que trazer benefícios aos seus moradores caso as mesmas sejam feitas de maneira não planejada.

Sendo assim, faz-se uso de estudos e técnicas de engenharia de tráfego e uso de simuladores de tráfego que permitem estimar de que forma tais modificaçôes influenciariam na região estudada, proporcionando assim, de forma direta, um incentivo à utilização do modal de veículos coletivos.

Objetiva-se então com este artigo mostrar o impacto da implantação dessas faixas exclusivas de ônibus em um estudo de caso da cidade de Curitiba, bem como mostrar a importância e vantagens em utilizar softwares de simulação de tráfego antes de efetivar alguma mudança no sistema viário, como é o caso desta pesquisa.

A literatura especializada traz alguns artifícios para otimizar o transporte coletivo de passageiros:

- Integração do transporte urbano com o objetivo de incentivar a utilização do mesmo nas cidades. Segundo [2], a forma mais clássica de integração é a baseada no conceito de um sistema tronco-alimentado. A outra forma de integração é a tarifária que, segundo [3], possibilita o aumento da acessibilidade às diferentes áreas da cidade atendidas pela rede de transporte, além de também criar uma maior liberdade de movimentação que permite que os próprios usuários racionalizem seus trajetos.

- Redução no tempo de parada de veículos. Conforme [4], o tempo ocioso despendido pelos veículos, considerando embarque e desembarque de passageiros, pode chegar a ordem de $50 \%$ dependendo do horário de pico analisado. A redução desse valor pode ser obtida com a utilização de faixas adicionais para ultrapassagem de veículos, a realizaçáo do embarque 
e desembarque no nível dos ônibus, a cobrança externa aos veículos ou a adoção de bilhetagem automática [2].

- Prioridade semafórica para o transporte coletivo. Outro redutor de velocidade dos ônibus é o tempo perdido em semáforos fechados que pode ser reduzido através de um correto dimensionamento dos cruzamentos. A prioridade pode ser diferenciada de duas formas: a forma convencional (conforme o horário e os dias da semana) e a forma automatizada (utilização de sensores nos cruzamentos que monitoram o tráfego).

Com o crescimento das cidades, as técnicas de Engenharia de Tráfego tornaram-se necessárias para melhorar a fluidez dos centros urbanos. Tem como finalidade, de acordo com o [5], permitir a segurança e eficiência para as vias através da ordenação dos movimentos, notificaçáo das leis ou ainda provendo alertas e orientações necessárias para uma boa operação dos elementos do tráfego. Tem-se como exemplo os controles de cruzamento, controle de velocidades ou determinação do uso das vias.

Para uma melhor aplicação dessas técnicas é necessário que se conheça as características e dados de cada situação estudada, obtidos com Estudos de Tráfego. [6] afirma que eles são os principais instrumentos utilizados por essa área da engenharia para atender as suas demandas, permitindo que se entenda a realidade e as projeçóes de tráfego da mobilidade de uma cidade. A melhor forma de se obter esses dados é através de pesquisas de tráfego, chamadas de Pesquisa de Contagem Volumétrica.

Sobre os simuladores, [7] os diferencia em 3 escalas de trabalho: microscópica, macroscópica e a mesoscópica. No mercado existe uma gama de softwares com suas diversas opçóes e objetivos, sendo que dois deles têm um maior destaque na área de transportes:

- AIMSUN. Software desenvolvido pela empresa TSS (Transport Simulation System) e utilizado para análises em nível microscópicas, porém também possui aplicação em nível mesoscópico. A empresa exemplifica alguns usos: otimização da prioridade de semaforização, linhas de BRT, viabilidade de faixas de tráfego, entre outros.

- PTV Vissim e PTV Visum. São dois softwares de simulação pertencentes ao grupo PTV Group. O software Visum é voltado para modelagem de transportes nas escalas macroscópica e mesoscópica, já o software Vissim é voltado para a simulação na escala microscópica. O Vissim pode ser utilizado para o desenvolvimento do planejamento de transportes, análise de capacidade, simulação de transporte público, entre outros. 


\section{Metodologia}

\subsection{Definiçáo do trecho a ser analisado}

A cidade utilizada no artigo é Curitiba e é importante analisar o sistema viário dela para determinação da via a ser estudada. Curitiba possui desde a década de 60 o seu desenvolvimento orientado pelo Uso do Solo e Sistema Viário. Para a implantação dos eixos estruturais e para a hierarquizaçáo das vias foi utilizado o Sistema Trinário que, conforme [8], é composto por uma via exclusiva central destinada ao transporte coletivo, duas vias de tráfego lento adjacentes à via exclusiva, e duas vias externas distantes uma quadra da via exclusiva, chamadas de tráfego rápido.

Atualmente, Curitiba conta com 5 eixos estruturais de transporte: norte, sul, leste, oeste e boqueirão. Como o enfoque do trabalho é o transporte coletivo de passageiros, foi identificada a via rápida do eixo estrutural que terá a maior quantidade de passageiros. $\mathrm{Na}$ Tabela 1 , pode-se observar o número de linhas e de passageiros que transitam em cada um dos eixos de acordo com [9]. Atenta-se para o fato de que o eixo leste não possui as vias rápidas externas, sendo assim não foi considerado no artigo. Com essa mesma tabela é possível identificar que o eixo a ser considerado é o sul.

Tabela 1 - Número de linhas de ônibus e volume de passageiros que transitam em cada eixo.

\begin{tabular}{ccccc}
\hline \multirow{2}{*}{ Eixo } & \multicolumn{2}{c}{ Número de linhas de ônibus } & \multicolumn{2}{c}{ Número de passageiros } \\
& Centro-Bairro & Bairro-Centro & Centro-Bairro & Bairro-Centro \\
\hline Boqueirão & 4 & 6 & 8.655 & 60.189 \\
Oeste & 19 & 14 & 116.115 & 137.843 \\
Norte & 7 & 13 & 90.291 & 88.174 \\
Sul & 34 & 48 & 137.594 & 174.494 \\
\hline
\end{tabular}

Com o objetivo de analisar somente o trecho crítico do eixo escolhido, é possível dividir o eixo em trechos conforme as linhas que passam em cada um deles nos dois sentidos, para assim determinar a quantidade de passageiros que transitam em cada trecho. No sentido centro - bairro o trecho crítico se localiza entre as ruas Itatiaia e Pedro Zagonel e no outro sentido o trecho mais carregado se localiza entre a Rua André João Gasparin e a Travessa Augusto Marach.

\subsection{Pesquisas de campo}

A contagem de veículos realizada é do tipo classificatória e direcional com o objetivo de identificar a quantidade de cada tipo de veículos que transitam na via e o movimento que cada um deles realiza nos cruzamentos. Conforme dados oficiais da [10] sobre contagem volumétrica de veículos obtidos com a fiscalização eletrônica, é determinado o horário pico de cada sentido do eixo estrutural 
estudado. Assim, as pesquisas de tráfego no sentido bairro - centro devem ser realizadas às 07 horas da manhã e no sentido centro - bairro devem ser realizadas entre 17 e 19 horas.

Além do horário, é necessário determinar os cruzamentos em que serão realizadas as contagens. No sentido bairro - centro, foram considerados os dois cruzamentos do trecho crítico mais dois (antes e depois do trecho crítico) para melhorar a qualidade da simulação. Já no sentido centro - bairro, o trecho crítico conta com 5 interseçôes semaforizadas sendo as mesmas utilizadas para a contagem dos veículos.

\subsection{Simulaçáo no software Vissim}

Com os dados obtidos nas pesquisas de contagem de volume de tráfego é possível simular a realidade do tráfego nas intersecçóes estudadas. Para tal estudo será utilizado o software Vissim oferecido para ser usado em conjunto com a Prefeitura Municipal de Curitiba.

$\mathrm{Na}$ simulação é necessária a configuração da base em que acontece a situação estudada, tais como as vias de tráfego, as conexóes, as áreas de conflito de veículos e a sinalização semafórica da regiáo. Além disso são necessários alguns dados de entrada relacionados a veículos, como, por exemplo, a quantidade de veículos que acessam a área de influência de estudo e os movimentos realizados pelos mesmos dentro dessa área. Com a base do sistema e os dados de entrada configurados, deve ser realizada a calibragem do mesmo através de parâmetros de calibragem fornecidos pela literatura, a depender do método utilizado.

Após a determinação de que o modelo está calibrado são realizadas todas as mudanças que se deseja estudar, no caso em questáo a adição de uma faixa exclusiva de ônibus em ambos os sentidos da via rápida do eixo estrutural. Para realizar a simulação em seguida, é indicado que sejam realizadas 15 simulações em sequência para se obterem resultados confiáveis da simulação. Após essa rodada de simulaçốes é possível obter os resultados com as modificações e realizar as análises pertinentes a tal estudo.

\section{Resultados}

Os primeiros resultados obtidos foram os fluxos de tráfego originados das pesquisas de contagem volumétrica classificatória e direcional, nos dois sentidos estudados. O simulador precisa que todos os veículos que entrem no sistema saiam dele e para isso foi necessário que se realizasse um balanceamento de todos os dados, sendo os mesmos representados na Figura 1, na qual o lado esquerdo é o sentido bairro-centro e o lado direito representa o sentido centro-bairro. 


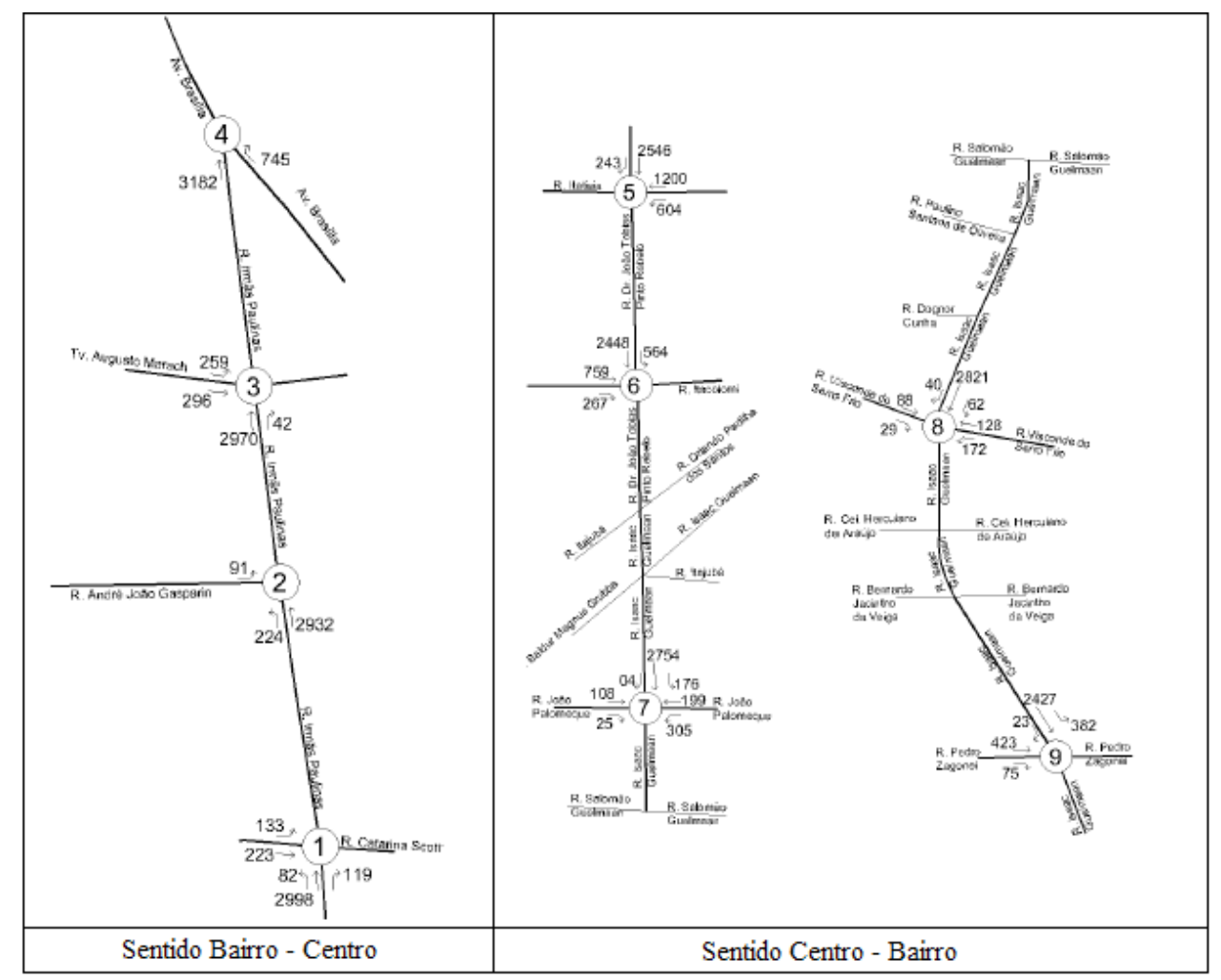

Figura 1 - Fluxograma de tráfego nos dois sentidos estudados.

Com os fluxogramas balanceados, é possível construir o modelo de tráfego e calibrá-lo de acordo com a realidade. Após isso, é possível simular a situação com faixa exclusiva desejada e obter os resultados que aconteceriam na prática após a implantação da faixa. Com os volumes de veículos da situação com faixa exclusiva e com os veículos da situação atual é possível fazer um comparativo entre os dois cenários.

$\mathrm{Na}$ Tabela 2, referente ao sentido bairro - centro, é possível observar a diferença percentual entre os movimentos de veículos particulares e de ônibus entre o cenário atual e o estudado. Nota-se que as percentagens negativas representam a diferença percentual de veículos retidos em cada cruzamento no novo cenário e as percentagens positivas representam que mais veículos conseguiram realizar tal movimento. Nota-se na mesma tabela que, com os resultados da simulação, até 7,94\% de veículos particulares ficam retidos em cada cruzamento para realizar o movimento principal quando da existência de faixa exclusiva de ônibus. Também observa-se que os ônibus náo sentiram efeito nenhum com a implantação da faixa, sendo nula a diferença percentual entre os dois cenários. 
Tabela 2 - diferença percentual entre os movimentos nos dois cenários no sentido bairro-centro.

\begin{tabular}{cccc}
\hline Cruzamento & Movimento & Veículos particulares & Ônibus \\
\hline & 1 & $-7,94 \%$ & $0 \%$ \\
2 & 3 & $-7,63 \%$ & - \\
1 & 4 & $-4,84 \%$ & $0 \%$ \\
& 6 & $0,00 \%$ & $0 \%$ \\
& 1 & $0,00 \%$ & - \\
\hline \multirow{2}{*}{2} & 3 & $-7,87 \%$ & $0 \%$ \\
& 6 & $-5,31 \%$ & $0 \%$ \\
& 1 & $0,00 \%$ & $0 \%$ \\
\hline \multirow{2}{*}{3} & 2 & $-7,50 \%$ & $0 \%$ \\
& 4 & $2,44 \%$ & - \\
& 6 & $0,00 \%$ & - \\
\hline
\end{tabular}

O simulador Vissim pode fornecer também os níveis de serviço de cada cruzamento. Na Tabela 3 é possível observar essa informação em cada cruzamento, tanto na situação atual como na estudada. Consegue-se observar também que a faixa exclusiva de ônibus não impactou de forma significativa os níveis de serviço, mesmo com a retenção de veículos observada anteriormente.

Tabela 3 - Níveis de serviço de cada cruzamento nos dois cenários no sentido bairro-centro.

\begin{tabular}{ccc}
\hline Cruzamento & Sem faixa exclusiva & Com faixa exclusiva \\
\hline 1 & $\mathrm{~B}$ & $\mathrm{C}$ \\
2 & $\mathrm{~A}$ & $\mathrm{~A}$ \\
3 & $\mathrm{~B}$ & $\mathrm{~B}$ \\
\hline
\end{tabular}

Da mesma forma que foi realizado com o sentido bairro - centro, é possível realizar algumas comparaçôes entre a situação sem a faixa exclusiva e a situação simulada com a faixa exclusiva no outro sentido. Na Tabela 4 tem-se a diferença percentual entre os movimentos nos dois cenários simulados no software Vissim. É possível observar que a retenção de veículos particulares nesses cruzamentos ainda é significativo para o movimento principal 1 de seguir em frente e para o movimento 2 de conversão à direita. Um exemplo crítico é o movimento 2 do cruzamento 7 em que quase todos os veículos ficaram retidos. Também é interessante notar que a faixa exclusiva de ônibus foi prejudicial para os próprios ônibus, tendo como exemplo o movimento 2 no cruzamento 5 que ficou inexistente. 
Tabela 4 - Diferença percentual entre os movimentos dos dois cenários no sentido centro-bairro.

\begin{tabular}{|c|c|c|c|}
\hline Cruzamento & Movimento & $\begin{array}{c}\text { Veículos } \\
\text { particulares }\end{array}$ & Ônibus \\
\hline \multirow{4}{*}{5} & 1 & $-2 \%$ & - \\
\hline & 2 & $-2 \%$ & $-100 \%$ \\
\hline & 4 & $-2 \%$ & - \\
\hline & 6 & $-2 \%$ & $-3 \%$ \\
\hline \multirow{4}{*}{6} & 1 & $-1 \%$ & $-3 \%$ \\
\hline & 3 & $-2 \%$ & - \\
\hline & 4 & $3 \%$ & - \\
\hline & 5 & $6 \%$ & - \\
\hline \multirow{7}{*}{7} & 1 & $0 \%$ & $-3 \%$ \\
\hline & 2 & $-97 \%$ & - \\
\hline & 3 & $-2 \%$ & - \\
\hline & 4 & $1 \%$ & - \\
\hline & 6 & $0 \%$ & - \\
\hline & 7 & $0 \%$ & - \\
\hline & 8 & $0 \%$ & - \\
\hline \multirow{7}{*}{8} & 1 & $-1 \%$ & $-3 \%$ \\
\hline & 2 & $0 \%$ & - \\
\hline & 3 & $3 \%$ & - \\
\hline & 4 & $0 \%$ & - \\
\hline & 6 & $0 \%$ & - \\
\hline & 7 & $0 \%$ & - \\
\hline & 8 & $0 \%$ & - \\
\hline \multirow{5}{*}{9} & 1 & $0 \%$ & $0 \%$ \\
\hline & 2 & $-60 \%$ & - \\
\hline & 3 & $-1 \%$ & $0 \%$ \\
\hline & 7 & $0 \%$ & - \\
\hline & 8 & $0 \%$ & - \\
\hline
\end{tabular}

$\mathrm{Na}$ Tabela 5, pode-se observar os níveis de serviço obtidos com o software Vissim para os dois cenários no sentido em questão. Nesse caso, observa-se uma diferença entre os níveis de serviço em dois cruzamentos do cenário estudado, a qual está de acordo com o observado na Tabela 4 em que, na simulação da faixa exclusiva, foram observados carros que ficaram retidos nesses cruzamentos. 
Tabela 5 - Níveis de serviço de cada cruzamento nos dois cenários no sentido centro-bairro.

\begin{tabular}{ccc}
\hline Cruzamento & Sem faixa exclusiva & Com faixa exclusiva \\
\hline 5 & $\mathrm{C}$ & $\mathrm{C}$ \\
6 & $\mathrm{~B}$ & $\mathrm{C}$ \\
7 & $\mathrm{~B}$ & $\mathrm{C}$ \\
8 & $\mathrm{~B}$ & $\mathrm{~B}$ \\
9 & $\mathrm{~A}$ & $\mathrm{~A}$ \\
\hline
\end{tabular}

\section{Conclusóes}

Com uma correta coleta de dados, modelagem da situação e simulação desejada é possível simular as opções previstas em projeto antes de serem executadas na realidade, evitando mudanças desnecessárias no sistema viário. Além disso, no que tange recursos necessários aos projetos de mobilidade, tem-se um melhor uso de recursos financeiros e humanos para os mesmos. Sendo assim, notam-se os simuladores como uma importante ferramenta de suporte a decisóes de modificaçóes significativas no meio urbano.

A coleta de dados em campo para este estudo foi necessária para observar que a capacidade da via rápida de Curitiba já está de certa forma saturada, vendo a necessidade de se pensar em uma mobilidade mais sustentável, através do transporte a pé, por bicicletas ou por veículos coletivos.

Como foi observado nas análises, não podem ser constatadas melhoras com a aplicação das faixas exclusivas nos dois sentidos no que se refere a número de veículos e/ou níveis de serviços dos cruzamentos. Isso ocorreu no sentido bairro - centro devido ao atual nível de serviço que não prejudica de certa forma os veículos coletivos e também devido a alocação do trânsito de veículos particulares de 4 faixas para 3. Já no sentido centro - bairro, porém, tem-se um nível de serviço mais crítico, o que já seria suficiente para influenciar positivamente o transporte coletivo e negativamente os veículos particulares. Tem-se como contradição que com a alocação integral dos veículos coletivos na faixa exclusiva gerou-se um congestionamento na própria faixa retendo alguns veículos que poderiam completar o movimento.

Ressalta-se que os resultados apresentados estão diretamente ligados a metodologia escolhida, que visou analisar os trechos com a maior quantidade de usuários do transporte coletivo. A realização de uma análise com outra metodologia poderia indicar a existência de alguns trechos mais críticos dependendo do parâmetro de comparação. Alguma outra metodologia também poderia indicar a necessidade da faixa exclusiva fragmentada em determinados trechos, ou somente uma que funcione em horários críticos. 


\section{Referências}

[1] SUMP GUIDELINES. Developing and implementing a sustainable urban mobility plan. European Union, 2013.

[2] SEDU/PR, Secretaria Especial de Desenvolvimento Urbano da Presidência da República. Relatório de Prioridade para o Transporte Coletivo Urbano. 2002. Disponível em: <http://www.fetranspordocs.com.br/ downloads/09PrioridadeTransporteColetivoUrbano.pdf>. Acesso em: 21 abr. 2016.

[3] CADAVAL, M. Desafios da integração tarifária. Cadernos Técnicos ANTP, São Paulo, SP, p. 99-107, 1 fev. 2007.

[4] CNT, COMPANHIA DE ENGENHARIA DE TRÁFEGO. Pesquisa CNT: Passageiros. Corredores de Transporte. Avaliaçáo dos corredores de Transporte por ônibus no Brasil. Brasília, CNT, maio 2002.

[5] U.S. DEPARTMENT OF TRANSPORTATION. Manual on uniform traffic control devices. Estados Unidos, 2003

[6] DNIT/IPR. Manual de Estudos de Tráfego. Publicação 723 IPR - Instituto de Pesquisas Rodoviárias. 384 p. Rio de Janeiro/RJ. 2006.

[7] BOGO, Rudinei Luiz; GRAMANI, Liliana Madalena; KAVISKI, Eloy. Modeling the flow of vehicles by the macroscopic theory. Rev. Bras. Ensino Fís., São Paulo, v.37, n. 1, 1301, mar. 2015.

[8] IPPUC. História do planejamento urbano de Curitiba. Disponível em: <http://www.ippuc.org.br/>. Acesso em: 26 ago. 2016.

[9] URBS. Estatístico de transporte coletivo de Curitiba. Curitiba/PR. 2016.

[10] SETRAN, Secretária de Trânsito. Fiscalizaçáo eletrônica. 2016. Disponível em: <http://www.setran.curitiba. pr.gov.br/servicos/fiscalizacao-eletronica>. Acesso em: 8 ago. 2016. 